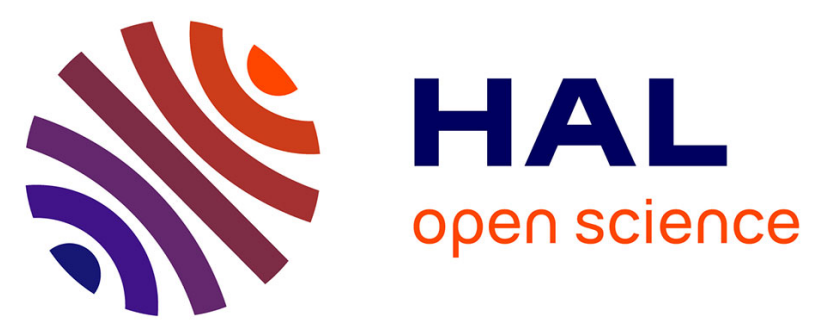

\title{
Utilité de la carte conceptuelle dans le suivi éducatif de patients au sein d'un pôle de prévention et d'éducation du patient
}

Claire Marchand, Béatrice Amory-rébérot, Marie van Der Schueren Etévé, Nathalie Proust, Laurence Ruelle, Jean-Marc Taupin, Rémi Gagnayre

\section{To cite this version:}

Claire Marchand, Béatrice Amory-rébérot, Marie van Der Schueren Etévé, Nathalie Proust, Laurence Ruelle, et al.. Utilité de la carte conceptuelle dans le suivi éducatif de patients au sein d'un pôle de prévention et d'éducation du patient. Éducation thérapeutique du patient / Therapeutic patient education , 2012, 4 (1), pp.31 - 40. 10.1051/tpe/2012002 . hal-01680922

\section{HAL Id: hal-01680922 \\ https://sorbonne-paris-nord.hal.science/hal-01680922}

Submitted on 11 Jan 2018

HAL is a multi-disciplinary open access archive for the deposit and dissemination of scientific research documents, whether they are published or not. The documents may come from teaching and research institutions in France or abroad, or from public or private research centers.
L'archive ouverte pluridisciplinaire HAL, est destinée au dépôt et à la diffusion de documents scientifiques de niveau recherche, publiés ou non, émanant des établissements d'enseignement et de recherche français ou étrangers, des laboratoires publics ou privés. 


\title{
Article original/Original article
}

\section{Utilité de la carte conceptuelle dans le suivi éducatif de patients au sein d'un pôle de prévention et d'éducation du patient}

\author{
Claire Marchand ${ }^{1}$, Béatrice Amory-Rébérot ${ }^{2}$, Marie Van der Schueren Etévé ${ }^{2}$, Nathalie Proust ${ }^{2}$, \\ Laurence Ruelle $^{2}$, Jean-Marc Taupin ${ }^{2}$, Rémi Gagnayre ${ }^{1}$ \\ ${ }^{1}$ Laboratoire de Pédagogie de la Santé, UPRES 3412, Université Paris 13, Bobigny, France \\ ${ }^{2}$ Pôle de Prévention et d'Éducation du patient de Soissons, Service éducation thérapeutique, Centre hospitalier Marie Sklodowska-Curie, \\ Soissons, France
}

(Reçu le 8 février 2012, accepté le 12 mars 2012)

\begin{abstract}
Résumé - Introduction : Dans le cadre du suivi éducatif à long terme des patients atteints de maladie chronique, les techniques éducatives utilisées pour analyser les besoins des patients sont peu formalisées. Objectif : Explorer l'intérêt pédagogique d'utiliser de la technique de la carte conceptuelle dans le cadre du suivi éducatif de patients porteurs de risques cardiovasculaire au sein d'un Pôle de Prévention et d'Éducation du Patient. Méthode : Élaboration d'une carte conceptuelle et négociation d'un contrat d'éducation au cours d'un entretien auprès de 16 patients. Résultats : Les deux tiers des besoins exprimés par les patients après la carte conceptuelle sont nouveaux par rapport aux attentes énoncées avant la carte. Les besoins exprimés par 11 patients sont totalement ou en partie identiques à ceux identifiés par les soignants au cours de l'entretien. Pour 13 patients, tous les besoins exprimés par les patients sont reliés à un domaine d'expression retrouvé dans leur carte conceptuelle. Pour 14 patients, les contrats éducatifs négociés tiennent compte de leurs besoins. Conclusion : L'étude montre que la carte conceptuelle constitue une aide à l'identification des besoins des patients et leur prise en compte dans la négociation d'un contrat d'éducation thérapeutique. Elle permet d'inscrire le suivi éducatif entre la perspective du patient et les propositions des soignants.
\end{abstract}

Mots clés : suivi à long terme / éducation thérapeutique du patient / carte conceptuelle / besoins éducatifs / maladie chronique

\begin{abstract}
Advantages of concept mapping in the long-term educational follow-up of patients in a specific educational structure. Introduction: Within the framework for the long-term educational follow-up of patients with chronic disease, the methods used to identify patient educational needs are not well described. Objective: Investigate the educational advantages of using concept mapping to make decisions about the educational follow-up of patients with cardiovascular risk factors in a specific educational structure. Method: Elaboration of a concept map and negotiation of an educational contract during an interview with 16 patients. Results: Two-thirds of the needs expressed by the patients after the concept map are new compared with the expectations expressed before concept mapping. The needs of 11 patients are fully or partially similar compared with those identified by the caregiver. For 13 patients, all the needs expressed by the patients are connected with a knowledge field found in their concept map. For 14 patients, the negotiated educational contracts take into account needs expressed by the patients. Conclusion: The study shows that concept mapping might help to identify patients' educational needs as well as to negotiate a therapeutic educational contract. It allows this followup to meet the patient's needs and the caregiver's educational objectives.
\end{abstract}

Key words: long-term follow up / patient therapeutic education / concept mapping / educational needs / chronic disease

\footnotetext{
${ }^{\star}$ Correspondance : Laboratoire de Pédagogie de la Santé, UPRES 3412, Université Paris 13, 74 rue Marcel Cochin, 93017 Bobigny Cedex, France, Claire.marchand@univ-paris13.fr
} 


\section{Introduction}

L'éducation thérapeutique fait partie intégrante de la prise en charge des patients atteints de maladie chronique. Elle constitue un processus continu intégré au soin, qui comprend différentes phases : une éducation initiale, qui fait suite au diagnostic médical ou une période de vie avec la maladie sans prise en charge éducative, et une période de suivi régulier ou de reprise éducative si le patient présente des difficultés particulières [1]. Lors de ce suivi éducatif, il est nécessaire de faire le point avec le patient sur l'évolution de ses compétences d'auto-soins et d'adaptation à la maladie [2] et sur les difficultés qu'il rencontre dans leur application au quotidien, ainsi que d'identifier de nouveaux besoins éducatifs le cas échéant. Un besoin est défini par l'écart existant entre une situation réelle, ou perçue comme telle, et une situation souhaitable. Un besoin éducatif correspond donc à ce que le patient devrait apprendre pour lui permettre de réduire cet écart et résoudre, par exemple, un problème de santé [3]. Un besoin peut être exprimé ou non, conscient ou non. Quand il est exprimé par le patient dans le cadre d'une éducation thérapeutique, il constitue une attente de sa part vis-à-vis des soignants. Le terme d'attente est employé ici comme synonyme de demande, désir ou souhait (dictionnaire le Petit Robert, 2003).

Dans le cadre des programmes d'éducation thérapeutique, les méthodes utilisées pour le suivi éducatif sont peu formalisées, en particulier celles permettant d'identifier les nouveaux besoins éducatifs du patient. En général, un bilan est réalisé au cours d'un entretien constituant parfois un nouveau diagnostic éducatif. Parfois, une liste de questions permet de réaliser un bilan rapide des connaissances d'un patient à la suite d'un incident par exemple [4,5]. Différentes techniques peuvent être utilisées pour aider à l'expression des besoins lors d'un diagnostic éducatif, par exemple : le dessin [6], le génogramme [7], le photolanguage [8]. De même, l'intérêt d'utiliser les cartes conceptuelles en éducation thérapeutique et principalement au cours d'un diagnostic éducatif a été étudié [9].

Nous nous posons la question de l'utilité de la carte conceptuelle lors d'un suivi éducatif tel que défini par la HAS [1].

À partir d'une expression accompagnée et libre du patient, la carte conceptuelle permet au soignant/éducateur d'identifier les connaissances et expériences acquises par le patient, ses croyances, ses représentations, ses connaissances erronées ou encore ses lacunes [10]. De plus, la réalisation d'une carte conceptuelle au cours d'un entretien avec le patient constitue un temps d'apprentissage dans la mesure où elle lui permet une prise de conscience de ses propres connaissances [10].

Elle facilite l'orientation des activités d'éducation thérapeutique par la définition d'objectifs d'éducation personnalisés. Elle est aussi parfois utilisée dans le cadre de consultation en médecine libérale, comme une étape préparatoire à la consultation [11]. Dans ce cas, le patient élabore lui-même sa carte avant la consultation. Le médecin s'appuie ensuite sur la carte pour mener sa consultation. La carte conceptuelle constituerait donc, à la fois pour les patients et pour les soignants, un outil au service de la recherche d'un accord sur les objectifs éducatifs, formalisé par un contrat d'éducation [12].

La carte conceptuelle est utilisée dans le cadre d'un suivi éducatif au sein du Pôle de Prévention et d'Éducation du Patient (PPEP) de Soissons qui fait partie des huit PPEP de la région picarde. Les missions de ces structures ont été précisées lors de l'élaboration d'un cahier des charges des activités des PPEP [13]. Le PPEP de Soissons a commencé son activité en septembre 2000, il s'est structuré en service d'éducation thérapeutique en 2004 [14]. Il accueille les patients présentant un facteur de risque cardiovasculaire modifiable tel que l'hypertension artérielle (HTA), le diabète, la dyslipidémie, le tabac ou ayant déjà une complication vasculaire telle qu'une cardiopathie ischémique, un accident vasculaire cérébral (AVC), une artériopathie des membres inférieurs, les patients souffrant d'une insuffisance cardiaque et ceux sous traitements anticoagulants. Deux programmes éducatifs sont proposés au titre de la loi Hôpital Patient Santé Territoire (HPST) : « maladie cardio-vasculaire » et « diabète » avec des activités communes aux deux programmes telles que la nutrition et l'activité physique. Ces programmes débutent après la réalisation du diagnostic éducatif et l'établissement d'un contrat éducatif. Les séances éducatives peuvent être réalisées en groupe ou en individuel, et se déclinent en une ou quatre parties en fonction de la thématique. Ces activités sont renforcées par des séances de suivi éducatif qui permettent d'évaluer avec le patient ce qu'il a compris, la façon dont il s'est approprié le contenu, le met en pratique dans son quotidien et les difficultés qu'il a rencontrées. Une évaluation de ses compétences est réalisée avant, à la fin et à distance du programme d'ETP.

Cet article a pour objectif d'explorer l'intérêt de l'utilisation de la technique de la carte conceptuelle dans le cadre d'un suivi éducatif pour aider le patient et les soignants/éducateurs à identifier de nouveaux besoins éducatifs et par conséquence inscrire le suivi éducatif dans un équilibre entre la perspective du patient et l'orientation éducative donnée par le soignant

\section{Matériel et méthode}

\subsection{Public}

Les patients inclus dans l'étude étaient des patients suivis au sein du PPEP, quelle que soit la pathologie. Ils devaient avoir suivi une éducation initiale, c'est-à-dire au moins trois séances d'éducation thérapeutique comprenant : un diagnostic éducatif, un (ou plusieurs) ateliers, et une séance de suivi à 6 mois. À l'issue de cette séance de suivi à 6 mois, les patients ont été invités à participer à un entretien individuel afin de faire le point sur leur santé et d'identifier leurs besoins éducatifs. Le 
rendez-vous était proposé aux patients soit à la fin de la séance, soit par contact téléphonique avec la secrétaire. Les patients étaient informés que cette évaluation se faisait dans le cadre d'une étude ; ils étaient libres d'accepter ou de refuser.

\subsection{Outil}

Les patients recrutés ont participé à des entretiens individuels réalisés par trois des membres de l'équipe soignante du Pôle : une infirmière, une diététicienne et un médecin. Cette équipe a été formée spécifiquement aux entretiens permettant d'élaborer des cartes conceptuelles.

Plusieurs étapes ont structuré ces entretiens :

- Étape 1. Introduction de l'entretien et identification des attentes initiales : demander au patient ses attentes par rapport à cette nouvelle rencontre (notées par le soignant) ; présentation au patient du déroulement de l'entretien et obtention de son accord pour l'enregistrement.

- Étape 2. Élaboration d'une carte conceptuelle à partir du concept central «ma santé aujourd'hui » inscrit au milieu d'une feuille A3. Le patient est invité à exprimer deux ou trois mots qui lui viennent immédiatement en lien avec le concept central (chaque concept est numéroté par ordre d'expression). Puis, la carte est élaborée progressivement par le soignant à partir des expressions des patients. La technique d'entretien utilisée vise à aider le patient dans l'explicitation de ses connaissances, représentations, expériences, etc. [10]. À la fin de l'entretien, le soignant relit la carte conceptuelle avec le patient pour s'assurer de la conformité des informations recueillies aux propos du patient et pour lui donner la possibilité de modifier ou compléter certaines informations s'il le souhaite.

- Étape 3. Identification des besoins éducatifs. Une fois la carte conceptuelle élaborée, la question suivante est posée au patient : dans tout ce que vous m'avez dit sur quoi souhaiteriez-vous travailler ? Pendant que le patient réfléchit à sa réponse, le soignant inscrit sur un papier les besoins qu'il a identifiés pour le patient. Le soignant note ensuite les besoins exprimés par le patient.

- Étape 4. Établissement d'un contrat d'éducation. Le patient et le soignant échangent sur les besoins identifiés, les buts à atteindre, et se mettent d' accord sur un contrat d'éducation thérapeutique.

Pour chaque patient, une fiche a été complétée, comprenant :

- Les données d'identification et médicales du patient;

- Les besoins éducatifs identifiés lors du diagnostic d'éducation à l'entrée du patient dans la structure et inscrits dans le dossier d'éducation du patient ;

- Les attentes du patient au début de l'entretien ;

- Les besoins du patient et ceux identifiés par le soignant après la réalisation de la carte conceptuelle ;

- Les éléments du contrat d'éducation thérapeutique à l'issue de l'entretien.

\subsection{Analyse}

Les cartes conceptuelles réalisées ont été retranscrites à l'aide du logiciel Inspiration (Fig. 1). La justesse des informations recueillies dans les cartes conceptuelles a été vérifiée par l'écoute des enregistrements d'entretiens par un chercheur n'ayant pas réalisé les entretiens. Une première analyse a été réalisée sur les cartes conceptuelles afin d'identifier les domaines de connaissances des patients selon une méthodologie d'analyse publiée [10,15]. Les domaines de connaissances correspondent dans cette étude aux grandes thématiques (ou sujets) abordées par les patients. Une fois les domaines identifiés, chaque carte conceptuelle a été analysée afin d'identifier quels étaient les domaines abordés par les patients, qu'ils soient ou non très développés (domaines structurants (S) ou non structurants (NS)). Puis les analyses suivantes ont été réalisées afin de répondre aux questions posées par l'étude :

- Comparaison des attentes des patients en début d'entretien avec les objectifs du diagnostic éducatif initial ;

- Évolution des attentes/besoins des patients à la suite de la réalisation des cartes conceptuelles ;

- Concordance des attentes/besoins exprimés par les patients avant et après la carte conceptuelle (ABP) avec les besoins identifiés par les soignants (BS) ;

- Concordance des contrats d'éducation avec les attentes/ besoins identifiés par les patients (ABP) et les soignants (BS) ;

- Concordance des attentes/besoins des patients (ABP) avec les domaines de connaissances et les mots d'entrées dans la carte conceptuelle.

\section{Résultats}

\subsection{Description de la population}

Seize patients ( 8 femmes/8 hommes) ont participé à l'étude sur une période de 3 ans (entre septembre 2008 et juin 2011). La moyenne d'âge est de 65 ans (51 ans-75 ans). Tous sont retraités sauf deux encore en activité. Les patients sont en moyenne suivis au pôle depuis 23 mois ( 8 mois-98 mois), avec en moyenne 14 séances éducatives réalisées par patient (3-18). Douze patients ont une maladie artérielle athéromateuse (infarctus, angine de poitrine, etc.), cinq sont diabétiques, quatorze ont une dyslipidémie, dix ont une hypertension artérielle, sept sont ex-fumeurs, un est fumeur, l'IMC est en moyenne de 27 (20-38).

\subsection{Description des domaines abordés par les patients}

Le tableau I présente la fréquence des domaines retrouvés chez les 16 patients, ainsi que ceux les plus développés par les patients (domaine structurant: S). Un domaine est considéré comme développé lorsque plusieurs propositions y sont reliées. 


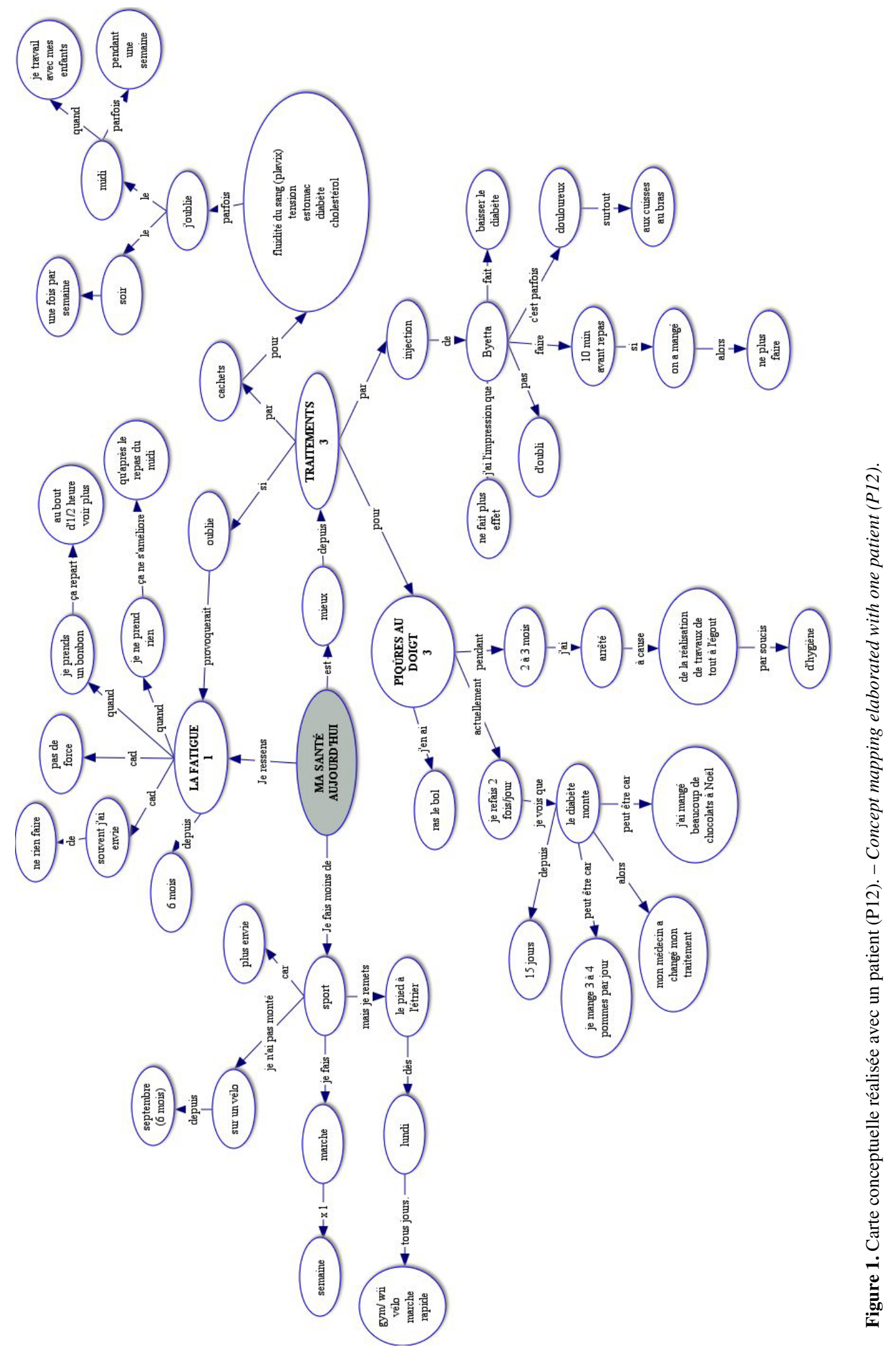


Tableau I. Fréquence des domaines de connaissances abordés par les patients dans leur carte conceptuelle $(n=16)$. - Distribution of patient's knowledge fields in the concept map.

\begin{tabular}{|l|c|c|}
\hline Domaine d'expression des patients & $\begin{array}{c}\text { Nombre de patients ayant abordé le } \\
\text { domaine }\end{array}$ & $\begin{array}{c}\text { Nombre de patient ayant développé le } \\
\text { domaine (S) }\end{array}$ \\
\hline Alimentation, équilibre alimentaire, régime & 14 & 5 \\
\hline Sport activité physique & 14 & 9 \\
\hline État général, psychologique, philosophie de vie & 14 & 9 \\
\hline Cholestérol, graisses saturées, insaturées & 14 & 6 \\
\hline Médicaments, traitements divers & 10 & 8 \\
\hline Maladie CV, douleur, physiopath, conduite à tenir & 9 & 1 \\
\hline Tabac & 8 & 2 \\
\hline Poids & 8 & 5 \\
\hline Autre maladie/autre problème & 7 & 0 \\
\hline HTA & 7 & 4 \\
\hline Diabète & 6 & 1 \\
\hline Émotion, sentiment relatif à la maladie & 6 & 0 \\
\hline Alcool & 5 & 2 \\
\hline Projection avenir & 5 & \\
\hline Suivi/bilan & 4 & \\
\hline Stress & 3 & \\
\hline Loisirs & 3 & \\
\hline Entourage & 3 & \\
\hline
\end{tabular}

Dix-huit domaines ont pu être identifiés. Huit domaines sont retrouvés dans les cartes de la moitié (ou plus) des patients. Quatre d'entre eux sont présents chez 2/3 des patients.

Les domaines qui sont uniquement évoqués (juste cités) ou faisant l'objet de peu de propositions (NS) chez les patients sont : l'HTA, l'alcool, le stress, les loisirs. Tous les autres domaines ont fait l'objet d'un développement important (S) chez au moins un patient. Les domaines les plus souvent développés par les patients sont (S) : l'alimentation, l'équilibre alimentaire, le régime (10 patients), l'état général tant physique que psychologique et philosophique ( 9 patients), la maladie cardiovasculaire, la douleur thoracique, les mécanismes physiopathologiques et les conduites à tenir (8 patients).

Les résultats suivants sont extraits du tableau II qui présente de façon synthétique les éléments pris en compte chez les patients et les soignants.

\subsection{Comparaison des attentes des patients en début d'entretien avec les objectifs du diagnostic éducatif initial}

Cinq patients sur $16(31 \%)$ n'expriment pas d'attente en début d'entretien. Les autres expriment des attentes identiques au diagnostic éducatif initial ( $9 / 16$ soit $56 \%$ ) en même temps que de nouvelles attentes (pour 2 de ces 9 patients). Deux patients expriment uniquement des attentes nouvelles. D'une manière générale, les attentes initiales relèvent de besoins éducatifs dans le sens ou une intervention spécifique favorisant l'apprentissage volontaire et conscient du patient pourrait permettre de les combler [12]. Ces attentes peuvent être d'ordre général : «valider les acquis » (P10), «refaire le point pour savoir si certains thèmes sont à approfondir » (P11); ou plus précises : «aide pour la gestion du diabète » $(\mathrm{P} 3)$, « apprendre à faire les piqures de Byetta ${ }^{\circledR}$ pour ne pas avoir mal » $(\mathrm{P} 12)$, «maintenirdes connaissances sur la maladie del'artère »(P5).

\section{4 Évolution des attentes/besoins des patients à la suite de la réalisation des cartes conceptuelles}

Trente besoins (B) sont exprimés par 14 des 16 patients à l'issue de la carte conceptuelle. Les deux tiers de ces besoins (20/30) sont nouveaux ou apportent des précisions aux attentes exprimées avant la réalisation de la carte conceptuelle.

\subsection{Concordance des attentes/besoins exprimés par les patients avant et après la carte conceptuelle (ABP) avec les besoins identifiés par les soignants (BS)}

Les soignants ont identifiés un total de 27 besoins (BS) et les patients 36 attentes/besoins (ABP : considèrent les 16 attentes exprimées avant la carte et les 20 nouveaux besoins 


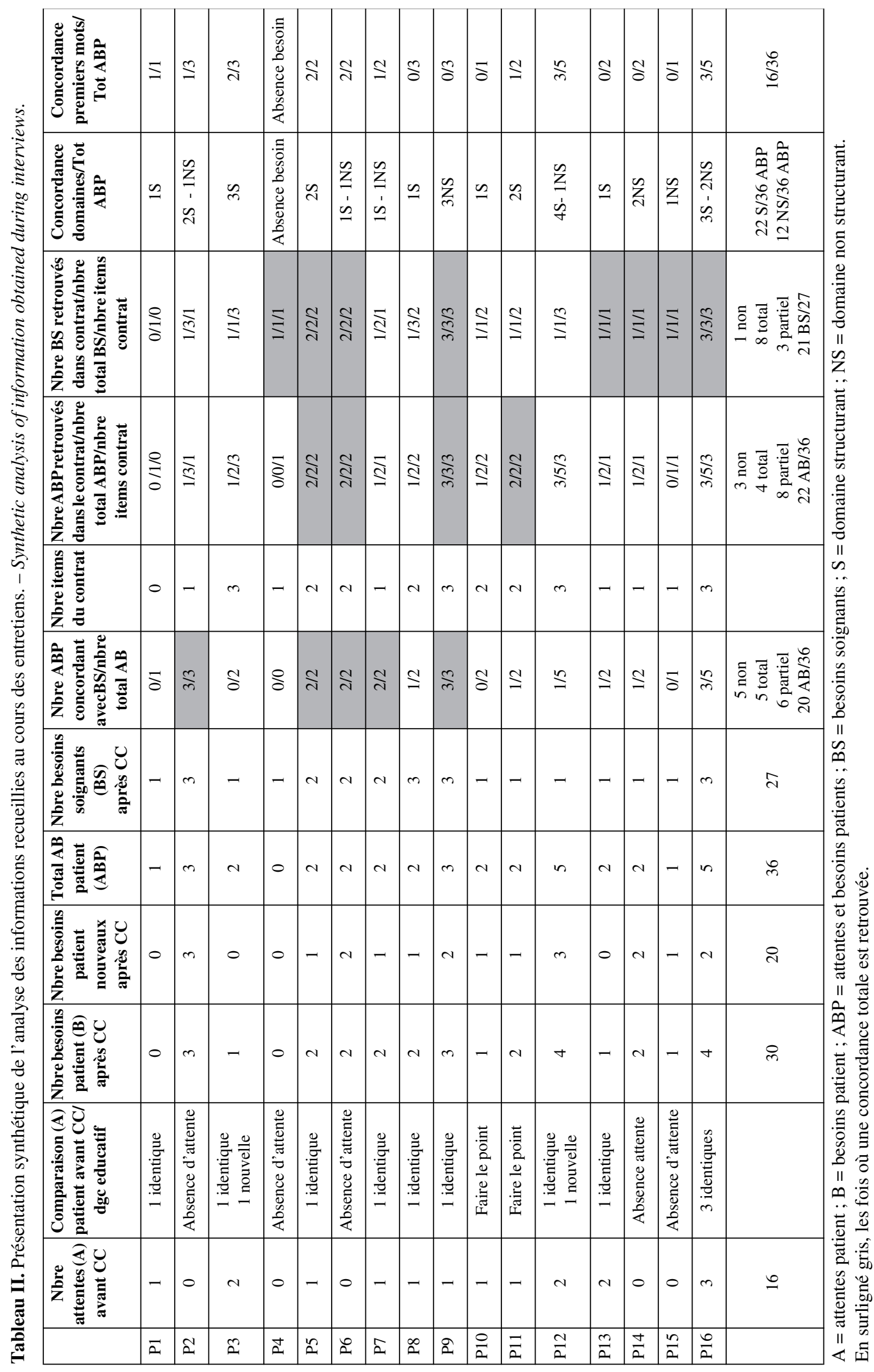


identifiés après la carte). Vingt ABP correspondent aux besoins identifiés par les soignants (BS), soit 55,5\% des BP. Les ABP et BS concordent chez $11 / 16$ patients $(69 \%)$, complètement pour 5 d'entre eux et partiellement pour 6 patients. Pour 5 sur 16 patients (31\%), les ABP ne correspondent pas du tout aux besoins relevés pour eux par les soignants (BS).

\subsection{Concordance des contrats d'éducation avec attentes/besoins identifiés par les patients (ABP) et les soignants (BS)}

Vingt-deux attentes/besoins de patients sont retrouvés dans les contrats d'éducation (soit $61 \%$ des ABP exprimés) et 21 besoins de soignants (soit $77,7 \%$ des BS). Huit contrats d'éducation prennent en compte l'ensemble des besoins soignants identifiés pour les patients correspondants. Parmi ces contrats, quatre tiennent aussi compte de l'ensemble des attentes/besoins exprimés par les patients (P5, P6, P9, P11). On constate que pour deux patients ( $\mathrm{P} 4, \mathrm{P} 15)$, les contrats éducatifs, s'ils correspondent totalement aux besoins relevés par les soignants, ne tiennent pas compte des attentes/besoins exprimés par les patients. À noter que pour P1, bien qu'un besoin éducatif ait été relevé par le soignant, il a été décidé de ne pas faire de proposition au patient sous la forme d'un nouveau contrat d'éducation. En effet, ce patient a clairement exprimé son absence de besoin à la fin de la réalisation de la carte conceptuelle, même si en terme d'attente il s'était exprimé avant la carte par un constat sur son état de santé «diabète que a du mal à baisser malgréle nouveau traitement » $(\mathrm{P} 1)$. On peut donc considérer dans ce cas que le soignant a tenu compte du besoin (ou de l'absence de besoin) du patient. Pour les neuf autres patients, les contrats d'éducation comprennent une partie seulement des attentes/besoins initialement exprimés ces patients.

\subsection{Concordance des attentes/besoins (ABP) avec les domaines de connaissances et les mots d'entrées dans la carte conceptuelle}

Toutes les attentes/besoins (ABP) exprimés par les patients sont reliés à un domaine de connaissances de la carte conceptuelle sauf pour trois patients : P8 qui précise avoir des attentes sans rapport avec des besoins éducatifs, P4 qui n'a exprimé aucun besoin, et P13 chez qui un des besoins n'est pas retrouvé explicitement dans la carte conceptuelle. En effet, ce patient aborde l'alimentation en général dans sa carte conceptuelle (domaine structurant) mais n'évoque jamais les notions de cholestérol ou de graisses alors qu'un de ces besoins concernent spécifiquement cet aspect «des questions sur le graisses ». Les 2/3 de ces ABP (22/36 ABP) sont liés à des domaines de connaissances structurant les cartes conceptuelles des patients (S). Chez 9 patients sur 16 (56\%), au moins un des mots d'entrée dans la carte conceptuelle correspond à une attente ou un besoin du patient. Pour $7 / 16$ patients (44 \%), aucun des mots d'entrée ne correspond à un besoin.

\section{Discussion}

Bien que cette étude, de type exploratoire, porte sur un nombre restreint de patients, elle nous permet de dégager quelques éléments en faveur de l'utilisation des cartes conceptuelles dans le cadre d'un suivi éducatif sur le long terme des patients atteints de maladie chronique. Le petit nombre de patients inclus dans l'étude sur une période relativement longue est principalement lié à l'organisation des programmes au sein de la structure. En effet, les programmes d'ETP proposés aux patients comportent de nombreux ateliers (jusqu'à 16 ateliers pour certains patients). Devant cette offre conséquente, et les patients étant libres de leur choix, un certain nombre d'entre eux n'effectuent pas l'ensemble du parcours proposé et/ou ne viennent pas au bilan à 6 mois au cours duquel la proposition de participer à un suivi éducatif à long terme est faite.

À noter que les analyses portent uniquement sur les informations recueillies et retranscrites par les soignants sur un document prévu à cet effet au moment de l'entretien (la fiche patient). Elles s'appuient donc sur des données déclaratives sans prendre en compte par exemple la qualité de la relation et/ou de la négociation donnant lieu à une décision de suivi éducatif.

\subsection{La carte conceptuelle comme aide à l'identification des besoins des patients au cours de leur suivi éducatif}

La comparaison des attentes exprimées par les patients avant la réalisation de la carte conceptuelle avec les besoins qu'ils identifient après son élaboration témoigne de la capacité de la carte conceptuelle à faire émerger chez les patients de nouveaux besoins éducatifs. En effet, si plusieurs patients arrivent dans la structure avec des attentes qu'ils expriment d'emblée, les deux tiers des besoins déclarés après la carte conceptuelle (20 sur 30) ne constituaient pas des attentes initialement évoquées par les patients. Par ailleurs, 4 des 5 patients n'ayant exprimé aucune attente en début d'entretien identifient des besoins après la réalisation de leur carte conceptuelle. De leur côté, les soignants relèvent de nombreux besoins pour les patients lors de l'élaboration de la carte (même si ces besoins sont inférieurs en nombre aux attentes et besoins identifiés par les patients euxmêmes, respectivement $27 \mathrm{BS}$ et $36 \mathrm{ABP}$ ). Ces résultats montrent à quel point ce temps de parole guidée par l'éducateur lors de la réalisation d'une carte conceptuelle permet au patient de prendre le temps de se centrer sur lui-même et son état de santé et l'aide à préciser certains besoins non spontanément exprimés. Plusieurs études ont montré à quel point les besoins éducatifs identifiés par les patients pouvaient être différents de 
ceux repérés par les soignants [16-18]. Cette étude confirme qu'il existe toujours des différences entre soignants et patients. En effet, pour cinq patients les besoins relevés par les soignants ne concordent pas du tout avec ceux identifiés par ces mêmes patients, et pour six autres les correspondances ne sont que partielles. Dans ces derniers cas, il est possible que les besoins identifiés par les soignants aient primé sur ceux des patients pour des raisons de priorités thérapeutiques par exemple (objectifs de sécurité). Au final, que ce soit les besoins du soignant ou ceux $\mathrm{du}$ patient qui sont choisis au moment de la rencontre, la recherche d'un accord nécessite pour le soignant de concilier une double posture éducative et thérapeutique. Ces résultats renforcent l'importance de consacrer un temps spécifique dans la démarche éducative pour permettre aux différents acteurs (patient et soignant) d'identifier ces besoins éducatifs et d'échanger sur leurs différences et complémentarités. La carte conceptuelle apparaît dans cette étude comme une technique facilitant l'émergence de ces besoins.

Cette étude montre également que les attentes des patients revenant au pôle dans le cadre d'un suivi à long terme sont pour plus de la moitié des patients (9) très proches des besoins éducatifs identifiés lors de leur première visite au moment du diagnostic éducatif (seulement 2 attentes sur 16 sont nouvelles). Les attentes des patients relèvent pour une grande majorité des cas de besoins d'apprentissage. Deux attentes font référence à d'autres aspects de l'éducation : la recherche d'un soutien (P3), d'une motivation (P16). Ces résultats peuvent en partie s'expliquer par le fait que les patients connaissent très bien la structure qui les accueille et dans laquelle ils ont déjà suivi un certain nombre de séances d'éducation thérapeutique (14 ateliers en moyenne). Ils expriment ainsi des attentes en lien avec les ressources éducatives qu'ils savent trouver au pôle. En effet, selon la définition des attentes proposées par Raynal et Rieuner [19], les attentes sont construites par l'individu à partir de son vécu, de sa perception de l' autre et de la connaissance du monde dans lequel il évolue.

Bien que cette étude ne vise pas à caractériser la nature des besoins, ces résultats montrent à nouveau à quel point l'éducation thérapeutique s'inscrit dans la durée non seulement pour l'apprentissage de nouvelles compétences mais aussi et surtout pour le maintien à long terme des compétences acquises, voir la réassurance de leur acquisition comme l'indiquent ces 3 patients : « besoin de faire le point » $(\mathrm{P} 11)$, «valider des acquis » $(\mathrm{P} 10)$, «maintenir ses connaissances sur la maladie artérielle » (P5). Nos résultats vont dans le même sens qu'une étude récente sur les besoins éducatifs des personnes vivant avec le VIH depuis plus de 10 ans. Cette étude a montré qu'un certain nombre de besoins éducatifs normalement abordés dans le cadre de programme d'éducation thérapeutique initiale perduraient dans le temps, phénomène observé dans d'autres études [20].

\subsection{La carte conceptuelle comme aide à la négociation d'un contrat d'éducation thérapeutique}

Seulement $61 \%$ des attentes/besoins des patients sont retrouvés dans les contrats contre $78 \%$ des besoins identifiés par les soignants. Il semblerait donc que les contrats négociés entre les deux acteurs soient plus proches des besoins identifiés par les soignants que de ceux exprimés par les patients. Cependant, ces résultats sont à interpréter avec précautions. En effet, les attentes/besoins des patients étant plus nombreux, cela a pu nécessiter de la part des soignants et des patients de sélectionner des besoins prioritaires, qu'ils correspondent ou pas à des objectifs de sécurité pour le patient [4]. Ces négociations apparaissent de différentes manières: contrat comprenant l'ensemble des besoins soignants et patients (P5, P6,P9) ; accord sur un seul objectif de contrat alors que plusieurs besoins étaient identifiés de manière identique par le patient et le soignant (P2, P7); accord sur un seul objectif correspondant à un besoin identique patient et soignant, les autres objectifs exprimés par le patient ou le soignant n'étant pas pris en compte (P12, P13, P14, P16) ; prise en compte à la fois des besoins du patient et du soignant alors que ces besoins se sont révélés différents (P3, P8, P10, P11) ; accord sur un objectif d'éducation relevant uniquement d'un besoin identifié par le soignant $(\mathrm{P} 4$, $\mathrm{P} 15)$; absence de contrat en accord avec l'absence de besoin exprimé par le patient (P1). Par ailleurs, certains ABP ne relèvent pas directement de l'éducation thérapeutique, ce qui peut expliquer leur absence de traduction dans un contrat d'éducation. L'ensemble de ces négociations semble montrer que la carte conceptuelle, utilisée dans le cadre d'un suivi à long terme, constitue une technique pertinente pour engager une discussion entre le patient et le soignant dans le but de décider et planifier ensemble une éventuelle reprise éducative [1]. Ces différents contrats traduisent la diversité des négociations ayant suivies la réalisation de la carte conceptuelle et témoignent de la capacité du programme d'éducation thérapeutique à personnaliser l'offre de suivi éducatif. Il pourrait être intéressant, lors d'une prochaine étude, d'étudier plus précisément les négociations en jeu lors de ces suivis éducatifs.

\subsection{Intérêts spécifiques de la carte conceptuelle}

La technique de la carte conceptuelle s'apparente à l'outil d'aide à la négociation d'objectifs éducatifs élaboré et testé au sein du PPEP de Beauvais. Comme la carte conceptuelle, cet outil propose une phase d'élucidation des besoins et une phase de négociation d'un contrat d'éducation. Les intérêts et les limites pédagogiques de cet outil ont été décrits récemment [21]. Deux aspects le distinguent particulièrement de la technique de la carte conceptuelle. D'une part, il peut s'utiliser avec le patient et son entourage afin de tenir compte des interactions familiales, alors que la carte conceptuelle telle qu'elle est utilisée dans cette 
étude reste individuelle. D'autre part, l'identification des besoins et la négociation des objectifs d'éducation se font sur la base d'un référentiel de compétences et d'objectifs d'éducation correspondant aux besoins d'apprentissage les plus courants dans le domaine de la diététique chez les patients atteints de pathologie cardiovasculaire. Contrairement à cet outil, les objectifs d'éducation négociés à la suite de la réalisation d'une carte conceptuelle ne sont pas suggérés et s'appuient uniquement sur les besoins conscientisés et explicités spontanément au cours de l'entretien. Plusieurs auteurs considèrent que dans un suivi de qualité il est important de considérer le patient comme un expert de sa maladie [22]. Il est donc essentiel de lui permettre d'exprimer librement sa perception de santé. Partir du concept « ma santé aujourd'hui », nous a semblé suffisamment large pour permettre au patient d'exprimer sa propre perception de santé au moment de la rencontre et d'envisager ensuite avec lui un suivi éducatif personnalisé. L'analyse des domaines de connaissances retrouvés dans les cartes conceptuelles témoigne de la diversité et de l'étendue des connaissances et représentations des patients en lien avec leur santé au moment de l'entretien. Si d'une part les $2 / 3$ des attentes/besoins des patients sont liés à des domaines structurants (S) et que d'autre part pour un peu plus de la moitié des patients (9/16) un ou plusieurs mots d'entrée dans la carte correspondent à leurs besoins, il est important de noter que d'autres besoins se révèlent au fur et à mesure de l'élaboration de la carte sans être obligatoirement reliés à un domaine particulièrement développé (domaines dits NS). Ces derniers résultats soulignent à nouveau à quel point la verbalisation progressive de ses connaissances et représentations par le patient lors de la réalisation d'une carte conceptuelle, lui offre la possibilité de prendre conscience et d'expliciter ses propres besoins éducatifs. Il apparaît essentiel d'associer à la réalisation de la carte un temps de discussion et d'échange sur les besoins identifiés, ces besoins n'étant pas obligatoirement reliés à un domaine développé (domaine $\mathrm{S}$ ).

Enfin, quel que soit le niveau de concordance entre besoins et développement de la carte conceptuelle, il est nécessaire de souligner que l'explicitation par le patient de ses besoins peut être dépendante de la manière dont le soignant gère le déroulement de la séance, en particulier la qualité de son écoute et la pertinence de ses relances pour aider le patient à approfondir ses propos sans lui suggérer de réponses. Cette dernière remarque pointe l'importance pour le soignant qui souhaite utiliser la carte conceptuelle de se former aux techniques d'aide à l'explicitation utilisées dans ce type d'entretien.

\section{Conclusion}

Cette première expérimentation permet de dégager les intérêts qu'il pourrait y avoir à utiliser la technique de la carte conceptuelle dans le cadre du suivi à long terme d'un patient atteint d'une maladie chronique. L'activité d'élaboration de la carte conceptuelle au cours d'un entretien entre un patient et un soignant a permis à chacun des acteurs d'identifier des besoins éducatifs. Le patient prend progressivement conscience de ses besoins, tandis que le soignant, centré sur le patient, l'accompagne par un questionnement visant l'explicitation de ses propos. L'étude montre que la carte conceptuelle peut être un véritable support pour la négociation des contrats d'éducation dans le cadre d'un suivi éducatif, à condition que les soignants éducateurs soient formés à son utilisation.

\section{Bibliographie}

1. HAS / INPES. Guide méthodologique: structuration d'un programme d'éducation thérapeutique du patient dans le champ des maladies chroniques, 2007. http://www.has-sante.fr

2. Ivernois (d') JF, Gagnayre R, et les membres du groupe de travail de IPCEM. Compétences d'adaptation à la maladie du patient : une proposition. Educ Ther Patient/Ther Patient Educ 2011; 3:S201-S205.

3. Deccache A, Lavendhomme E. Information et éducation du patient. Des fondements aux méthodes. Bruxelles : De Boeck Université; 1989, 239 p.

4. Ivernois (d') JF, Gagnayre R. Apprendre à éduquer le patient, approche pédagogique. $2^{\mathrm{e}}$ éd. Paris : Maloine; 2004, 155 p.

5. Bolman C, Brug J, Bar F, Martinali J, van den Borne B. Long-term efficacy of a checklist to improve patient education in cardiology. Patient Educ Couns 2005; 56:240-248.

6. Ginières B. Gagnayre R. La représentation de l'asthme par l'enfant au travers du dessin de la santé : un outil pour la relation éducative. Éducation du Patient et Enjeux de Santé 2004; $22: 73-78$.

7. Juré E, Iguenane J, Toudounou A, Azondecon A, Gagnayre R. Utilité du génogramme dans l'éducation thérapeutique: une étude exploratoire auprès de parents d'enfants vivant avec le VIH/ sida au Bénin. Santé publique 2010; 22:11-22.

8. Baptiste A, Belisle C, Pechenart JM, Vacheret C. Photolangage. Une méthode pour communiquer en groupe par la photo. Paris : Les Éditions d'Organisation ; 1991.

9. Marchand C., La technique des cartes conceptuelles comme outil d'aide au diagnostic éducatif, (en ligne). 2005, http:// www.ipcem.org

10. Marchand C, d'Ivernois JF, Slama G, Assal JP, Hivon R. An analysis, using concept mapping, of diabetic patient's knowledge, before and after patient education. Medical Teacher 2002; 24:90-99.

11. Bonadiman L, Gagnayre R, Marchand C, Marcolongo R. Utilisation de la carte conceptuelle en consultation médicale. Éducation du Patient et Enjeux de Santé, 2006; 24:46-50.

12. Rébérot-Amory B., La carte conceptuelle : un outil au service de l'alliance thérapeutique. Quels avantages, quelles limites. Mémoire de Diplôme Universitaire, Université de Picardie, Jules Verne, 2006, $78 \mathrm{p}$. 
13. Brun MF, Gagnayre R, Libion F, Deccache A. Élaboration d'un cahier des charges d'activités : pôle de prévention et d'éducation de Picardie. Gestions Hospitalières 2006; 452:36-44.

14. Amory-Rébérot B, Taupin J-M, Van der Schueren-Etévé M, Proust N, Floury I, Tessin V. Réflexion sur l'exercice professionnel dans le domaine de l'éducation thérapeutique du patient. Soins 2007; 716:S19-S22.

15. Marchand C, Poitou C, Pinosa C, Harry I, Basdevant A, d'Ivernois JF. Using concept maps to describe the evolution in obese patients' knowledge and expectations following bariatric surgery. Educ Ther Patient/Ther Patient Educ 2009; 1:49-55.

16. Pennel MP, Szymczak V, Wojtasik-Condette G, Demory C, Gagnayre R. Analyse croisée des besoins d'éducation thérapeutique exprimés par les patients atteints de sclérodermie systémique et par leurs soignants d'un service hospitalier référent. Education du patient et enjeux de santé 2008; 26:5-11.

17. Ledey D, Metté C, Gagnayre R. Besoins et compétences des patients dialysés en centre dans la gestion de leur maladie et de leur traitement dans leur vie quotidienne : points de vue croisés entre les patients et les soignants. Éducation du patient et enjeux de santé 2006; 24:22-31.

18. Spiesser-Robelet L, Rouault A, Prot-Labarthe S, Bourdon O, Oury JF, Brion F, et al. Analyse des besoins éducatifs des femmes allaitantes vis-à-vis de la prise de médicaments. Educ Ther Patient/Ther Patient Educ 2010; 2:25-38.

19. Raynal F, Rieunier A. Pédagogie : dictionnaire des concepts clés. Apprentissage, formation et psychologie cognitive. Paris : EFS éditeur; 1997.

20. Communier A, Sadki R, Sommerlatte E, Gagnayre R. Quels besoins éducatifs pour des personnes vivant avec le VIH depuis plus de 10 ans? Educ Ther Patient/Ther Patient Educ 2010; 2:51-62.

21. Lecompte M, Virlan E, Paviot A, Gagnayre R. Proposition d'un outil d'aide à la négociation d'objectifs éducatifs pour le patient et son entourage : intérêts et limites pédagogiques. Educ Ther Patient/Ther Patient Educ 2011; 3:S301-S311.

22. Assal JP, Golay A. Le suivi à long terme des patients chroniques : les nouvelles dimensions du temps thérapeutique. Rev Med Suisse 2008; 23-53. 